PREFACE

\title{
Essays on OR in ALIO country members (part 1)
}

\author{
Laureano F. Escudero $^{1} \cdot$ Antonio Alonso-Ayuso $^{1} \cdot$ Nelson Maculan $^{2}$
}

Published online: 22 September 2020

○ Sociedad de Estadística e Investigación Operativa 2020

Mathematics Subject Classification 90B90 - 90B06 · 90C06 · 90C08 · 90C46 · $90 \mathrm{C} 90$

The Spanish Sociedad de Estadística e Investigación-Operativa, SEIO, devotes two volumes of its journal TOP (co-guest-edited by Antonio Alonso-Ayuso, Laureano F. Escudero and Nelson Maculan) to present works by representative authors of ALIO member countries. ALIO (Asociación Latino-Iberoamericana de InvestigaciónOperativa), https://www.alio-online.org/, is a regional association within IFORS.

This volume is the first of the two ones, where selected peer-reviewed works are collected. Those works vary from a mixture of more theoretical and algorithmic developments in cooperative games, and nonlinear, combinatorics, integer, bilevel, stochastic and dynamic optimization ones to more applied works on problem solving in rank aggregation, discrete, competitive, economic and environmental location, cross-dock door assignment and truck scheduling, fleet management, demand response in power grids, offshore oil platform production, reliability in network topology design, and sustainable timber harvesting.

The aims of the works are as follows:

Short-term production of offshore oil platforms under uncertainty is studied in the paper "A stochastic optimization model for short-term production of offshore oil platforms with satellite wells using gas lift", by Carlos Gamboa, Thuener Silva, Davi Valladão, Bernardo K. Pagnoncelli, Tito Homem-de-Mello, Bruno Vieira, and Alex Teixeira. A steady-state two-stage stochastic model is proposed to maximize short-term production of offshore oil platforms. The first-stage injection level determines the production potential, while recourse actions ensure

Laureano F. Escudero

Laureano.escudero@urjc.es

Antonio Alonso-Ayuso

antonio.alonso@urjc.es

Nelson Maculan

maculan@cos.ufrj.br

1 Universidad Rey Juan Carlos, Madrid, Spain

2 Universidade Federal do Rio de Janeiro, Rio de Janeiro, Brazil 
capacity and platform constraints for each uncertainty realization (i.e., scenario). In particular, a concave approximation of the performance curve that incorporates uncertainty in the water cut and gas-oil ratio is developed.

The paper "Towards sustainable timber harvesting of homogeneous stands: dynamic programming in synergy with forest growth simulation", by Mario C. Lopez-Loces, Roger Z. Rios-Mercado, Oscar Alberto Aguirre-Calderon, and José Luis Gonzalez-Velarde, addresses the problem of maximizing the volume of wood harvested in a single-species stand over a period of time. To this end, a solution that combines dynamic programming and a single-tree forest growth simulator is developed, where the decision variable of the amount of wood to be harvested at each period is discretized. This ensures that the method finds a global optimal solution within the given discretization.

Ubaldo M. Garcia-Palomares proposes a non-monotone direct search method (NMDSM) that finds a stationary point of linearly constrained minimization problems in his contribution "Non-monotone derivative-free algorithm for solving optimization models with linear constraints: extensions for solving nonlinearly constrained models via exact penalty methods". He also considers possible extensions of NMDSM to non-linearly constrained problems via exact penalty function. A slightly modified algorithm is also proposed for satisfactorily solving a multi-batch multi-product plant that is modeled as a mixed integer nonlinear optimization problem.

Joshua L. Pulsipher and Victor M. Zavala propose a computational framework to optimize the reliability in network topology design of complex systems in their contribution "Measuring and optimizing system reliability: a stochastic programming approach". The approach uses a graph representation of the system that is subject to random failures of its components (nodes and edges). Under this setting, reliability is defined as the probability of finding a path between sources and sink nodes under random component failures. It is shown that this measure can be computed by solving a stochastic mixed-integer optimization model.

The linear ordering problem is studied by Javier Alcaraz, Eva M. Garcia-Nove, Mercedes Landete, and Juan F. Monge in "The linear ordering problem with clusters: a new partial Ranking"; in particular, the authors tackle the case in which the columns of the squared non-negative matrix in the highly combinatorial linear ordering problem belong to different clusters. The goal is to order the clusters by finding the permutation of rows and columns which maximizes the sum of superdiagonal values. A new approach is presented for the case when exactly one representative is chosen from each cluster.

The paper "On egalitarian values for cooperative games with a priori unions", by Jose Maria Alonso-Meijide, Julian Costa, Ignacio Garcia-Jurado, and Juan Carlos Goncalves-Dosantos, presents an extension of the equal division and the equal surplus division values for transferable utility cooperative games to the more general setup of transferable utility cooperative games with a priori unions. In the case of the equal surplus division three possible extensions are proposed, and axiomatic characterizations of the new values are also introduced.

Juan Di Mauro and Hugo D. Scolnik in "An augmented filled function for global nonlinear integer optimization" present an approach that does not depend on 
a particular local optimization method (as e.g., the steepest descent one for local search) for finding a global minima of nonlinear discrete functions, and a new discrete filled function. It has the useful property that a good continuous global optimization algorithm applied to it leads to an approximation of the solution of the nonlinear discrete problem. Numerical results are given showing the efficiency of the new approach.

In "Integrated cross-dock door assignment and truck scheduling with handling times", Sayed Ibrahim Sayed, Ivan Contreras, Juan A. Diaz, and Dolores E. Luna address an integrated cross-dock door assignment and truck scheduling problem. The authors simultaneously determine the assignment and scheduling of incoming trucks to inbound doors for unloading commodities and outgoing trucks to outbound doors for loading commodities after being consolidated according to their destinations. The objective is the minimization of the total time to process all trucks. Two formulations of the model are proposed as well as two hybrid metaheuristics for problem solving.

Real-time fleet management is a very interesting problem that is tackled by Javiera Barrera, Rodrigo Carrasco, and Eduardo Moreno in the paper "Real-time fleet management decision support system with security constraints". The authors present a fast and efficient decision support tool to help fleet managers oversee and control ore trucks, by allowing to work with real-life noisy GPS data in a mining setting. The main objective of the system is to help managers to conflict detection and resolution (i.e., to avoid interactions between ore trucks and personnel buses), one of the most critical security constraints in the case study under consideration, keeping a minimum security distance between the two at all times.

The paper "Economic and environmental location of logistics integration centers: the Brazilian soybean transportation", by Conrado V. Plaza, Vanessa de A. Guimarães, Glaydston Ribeiro, and Laura Bahiense, presents a study to locate logistics integration centers (LICs) by applying a capacitated multi-layer location-allocation mathematical optimization model, considering economic (transport and installation costs) and environmental criteria $\left(\mathrm{CO}_{2}\right.$ emissions). The proposed model is applied on scenarios that include information of the National Logistics and Transport Plan about soybean transport in Brazil.

The contribution by H.A. Eiselt and Vladimir Marianov, "Stability of utility functions and apportionment rules in location models" investigates how demand is apportioned to facilities by customers, given that one of a number of utility functions is applied, according to which the customer demand at the facilities is satisfied. A number of reasonable assumptions are formulated regarding the behavior of the utility functions after a scaling of their different parameters. The individual apportionment rules are examined so as to whether or not they satisfy these assumptions.

Enrique Domínguez and Alfredo Marín in "Discrete ordered median problem with induced order" extend the classical ordered median problem by considering a second weight that is associated to the distance from a customer to its closest facility. That weight is based on the distance from the customer to the closest facility of a different type. For the solution of the proposed model several integer programming formulations are built and computationally compared. 
Carlos Henggeler Antunes, Maria João Alves, and Billur Ecer present an interesting review on the use of bilevel optimization in problems that involve consumers' demand response arising in the power sector in "Bilevel optimization to deal with demand response in power grids: models, methods and challenges". The importance of demand response as a "dispatchable" resource in the evolution of power networks to smart grids is emphasized. The hierarchical nature of the interaction between decision-makers establishes bilevel optimization as an adequate approach to decision support.

Publisher's Note Springer Nature remains neutral with regard to jurisdictional claims in published maps and institutional affiliations. 